\title{
Framework of Intelligent Search Engine using Structured Data
}

\author{
Aman Patial \\ M.C.A (Student) \\ Lovely Professional \\ University, Phagwara
}

\author{
Rahul Kumar \\ M.C.A (Student) \\ Lovely Professional \\ University, Phagwara
}

\author{
Rohit Ohri \\ Assistant Professor \\ (Computer Application) \\ Lovely Professional \\ University, Phagwara
}

\begin{abstract}
In this study, the problem is faced by higher authorities and those person who don't know a little bit of sql query that's why they don't fetch the information from the structured data. This proposed algorithm first to gathering data with the help of user. Framework of intelligent search engine extract the information and displays on the front end. User can export the information in various format like pdf, doc, xml, excel etc. The results obtained from the intelligent search engine the information extraction are optimal.
\end{abstract}

\section{General Terms}

Intelligent Search Engine Using Structured data

\section{Keywords}

Full text search query in sql, Conversion in xml format, Export data, Microsoft rank in-built function

\section{INTRODUCTION}

Every search engine have search the data based upon some conditions at the front end or back end. Search engine uses the natural language which is entered by the user and perform some searching operation on the basis of some techniques like ranking algorithm are used is google search engine. Search engine is basically a software which uses some algorithms and in-built function to perform a search. For example, in windows operating system there is one search box for searching the data into the folder/directories. This is one of the best example why searching is more important in our daily life. Following section describes the basic concept of this research paper.

\subsection{Intelligent Search Engine Using Structured Data}

Data are to be extract by the input controller and it converts the data into one single format like excel, access, and tabular form of data are converted into xml sql database.

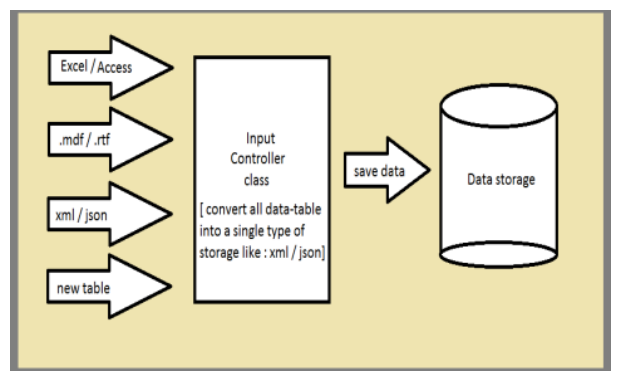

After extract the data storage algorithm are stored the data into database storage.

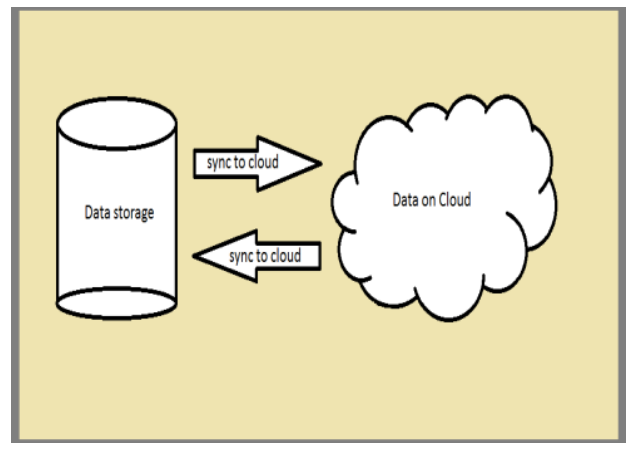

User interact with framework of intelligent search engine box. Searching algorithm generate the run time query side. The output controller generate the result and it can represent the data in various form.

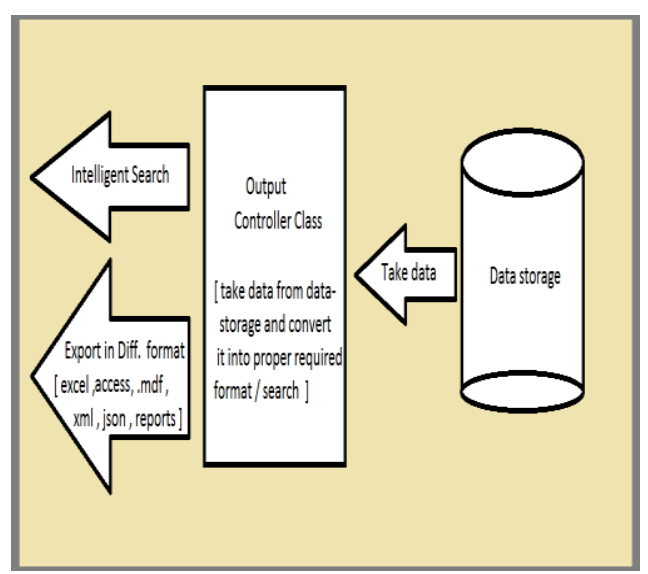

Therefore, it can download the result in various format (pdf, doc) with user requirement.

\subsection{Description of Problem}

They are proposed this research paper because they have seen that mostly the higher authorities don't know the sql query. If they want to search some data into the sql database than they can't do anything. That's why they have been implement the intelligent search engine using structured data with the help of c\# using front end and microsoft sql server database back end. They have solve the higher authority problem with the help of full-textsearch query. Higher authority enter the natural language in search box and full-text-search execution in the back end to search the data and show the result on viewer screen. 


\section{OBJECTIVE}

- Data conversion for input controller.

- Data storage algorithm.

- Searching algorithm.

- Generation of run time queries / joins / union etc.

- Intelligence feature while searching.

- Data (generated data) representation to end user.

- Data conversion for output controller.

- Full text search query in microsoft sql server.

\section{PROPOSED SOLUTION}

In this research paper they can solve the problem with the help of framework of intelligent search engine using structured data. In this framework higher authority searches some text with the help search engine. Therefore this text work as a full text search query which hit on to the sql xml database to retrieved the data. They perform search query on xml because it is light weight and full text search query run faster.

\subsection{Processing Of Natural Language Query}

This framework uses the natural language to search the data. Natural language are irregular and asymmetrical and natural language are based on un-formal language [11] How you can uses the natural language it's all depend upon the logic behind used it. Example google search engine uses the ranking algorithm which is very helpful for google search [6]. Figure-1 represents that how's natural language query work on the framework of intelligent search engine.

\section{Higher Authority}

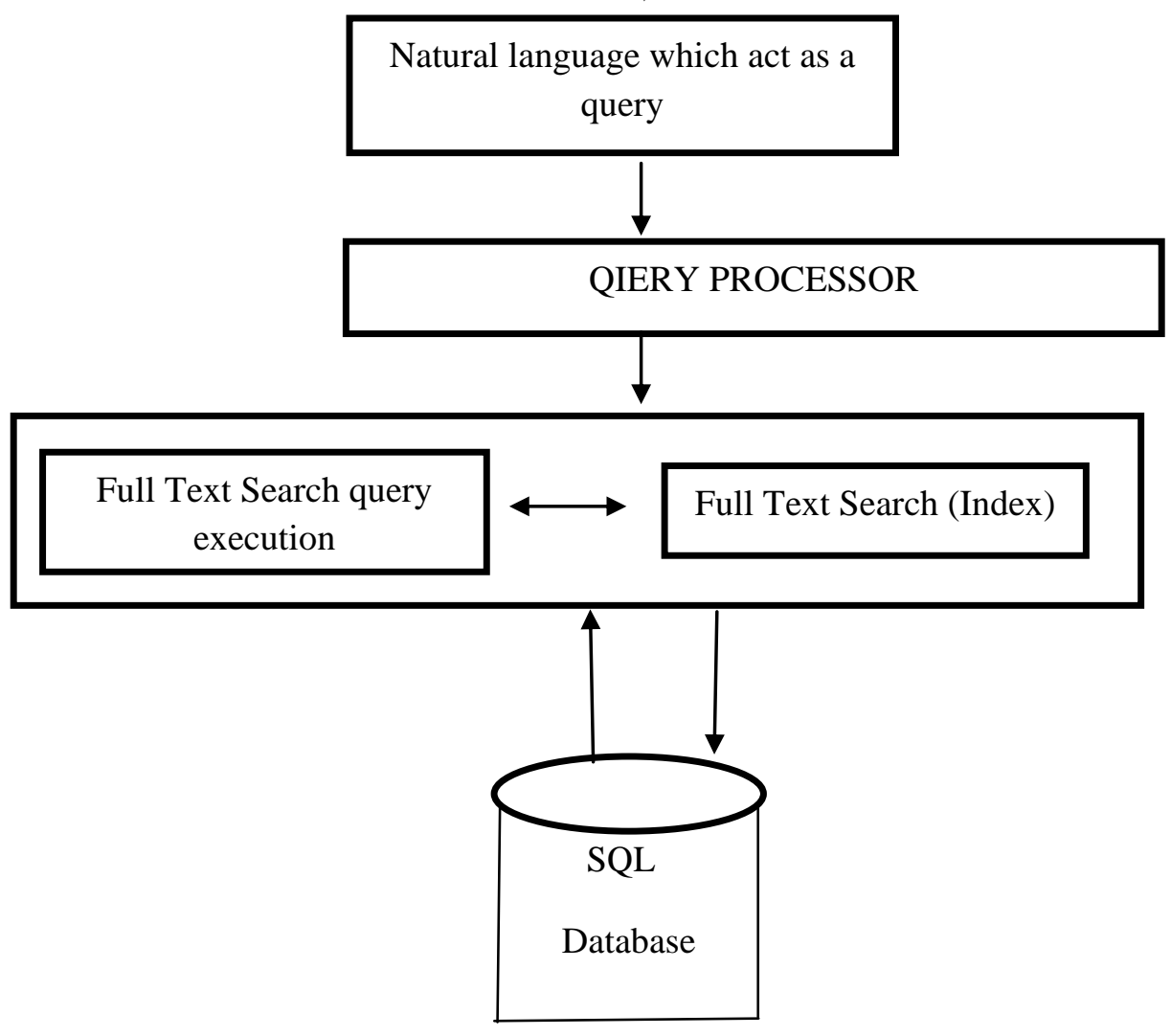

Figure- 1 


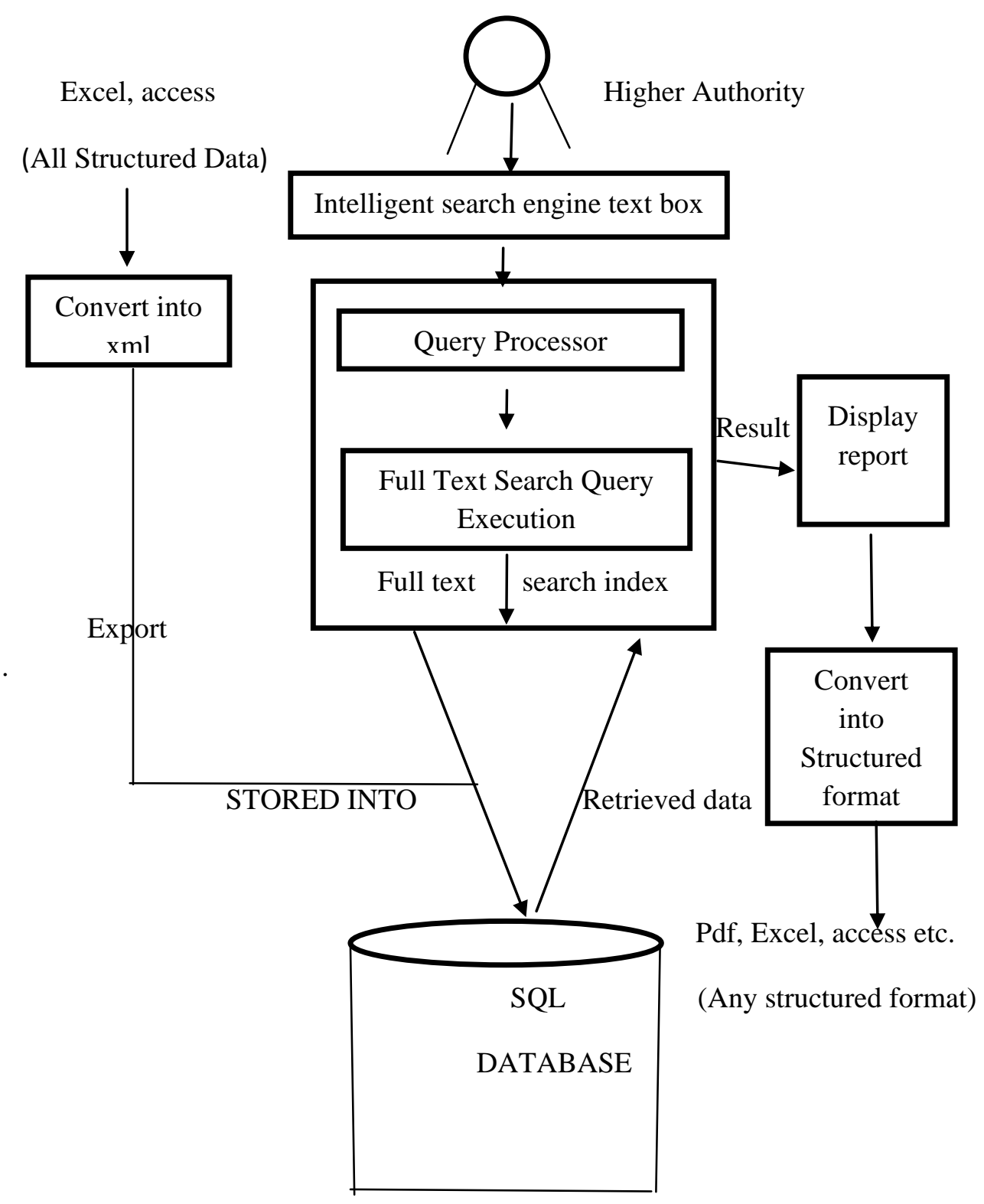

Figure- 2 proposed system architecture

\section{PROPOSED SYSTEM ARCHITECTURE}

Proposed system architecture are uses various task following as

- Convert the structured data into $\mathrm{xml}$ form with the help of convert class.

- Higher authority searches the data from search engine using $\mathrm{c} \#$ front end.

- Higher authority get some result from the sql database which showed the result some data representation.

- Full text search query hit on the sql database to get the result.

- Result will convert into any structured format like excel, access, pdf etc.
Figure-2 represents the framework of intelligent search engine system architecture.

\section{RESEARCH METHODOLOGY}

Using framework of intelligent search engine whenever the higher authority user wants to search something from the search engine so the search will be performed on the xml database with the help sql full text search query. The search query returns the data on to the viewer screen. This technique will return those record which satisfied the user requirement. This full text search query result also generated with the help of like operator in sql.

Comparison between sql full text search query and like operator are following as:-

Example: - using like operator in sql

USE test;

GO

SELECT x.FirstName, x.LastName, y.PhoneNumber 
FROM Person.PersonPhone AS y

INNER JOIN Person.Person AS $\mathrm{x}$

ON y.BusinessEntityID $=x \cdot$ BusinessEntityID

WHERE y.PhoneNumber LIKE '415\%'

GO

\section{Like query result}

Table -1 PersonPhone

\begin{tabular}{|l|l|l|}
\hline First name & Last name & Phone \\
\hline XYZ & JB & $415-555-124$ \\
\hline ABC & SM & $415-845-546$ \\
\hline PQR & UV & $415-545-844$ \\
\hline
\end{tabular}

Example: - using full text search query in sql

USE test

GO

SELECT BusinessEntityID, JobTitle

FROM HumanResources.Employee

WHERE FREETEXT (*, 'Marketing Assistant');

Full text search query result

Table -2 Employee

\begin{tabular}{|l|l|}
\hline BusinessEntityID & JobTitle \\
\hline 21 & $\begin{array}{l}\text { Marketing } \\
\text { specialist }\end{array}$ \\
\hline 25 & $\begin{array}{l}\text { Document control } \\
\text { assistant }\end{array}$ \\
\hline 27 & $\begin{array}{l}\text { Scheduling } \\
\text { assistant }\end{array}$ \\
\hline
\end{tabular}

In above you can see that like transact sql query search on the basis of character pattern. You can't use the like transact sql query for binary formatted data. sql full text search query against the unstructured text data is much faster than like query against the data [12].Full text query against the text data can take seconds to return the millions of rows whereas the like query against the same data can take minutes or large time. You can also use the boolean operator in full text search query using CONTAINS and CONTAINSTABLE function [13].

\section{RESULT AND DISCUSSION}

In this research paper the framework of intelligent search engine using structured data to implement this they can use $\mathrm{c \#}$ as a front end and microsoft sql server as a back end. They can analysis the performance of implementation part with the help of Jet Brains dot trace 6.1 software. The analysis of performance evaluation are following represented briefly.

\subsection{Performance Evaluation}

They have calculated the main thread performance call of our software framework of intelligent search engine using structured data. The following figure- 3 and figure- 4 describe the call performance of system code, garbage collection, user code etc.

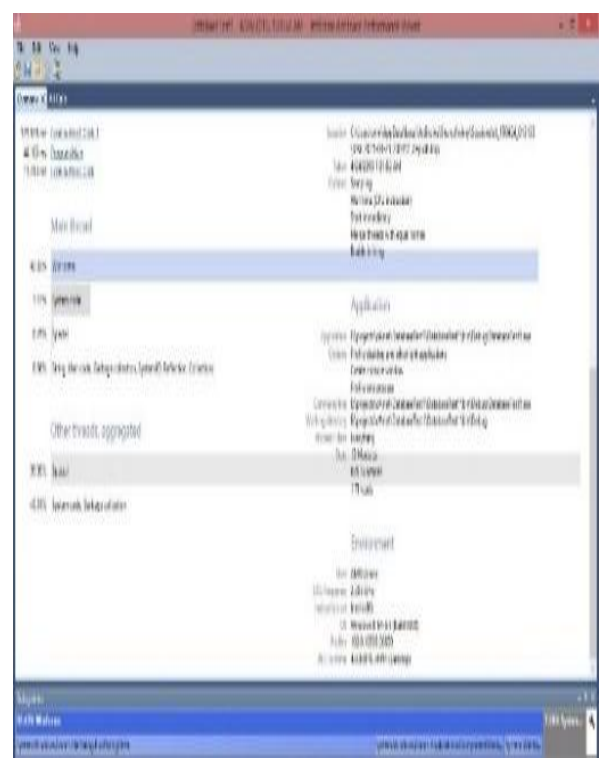

Figure- 3 Main report of performance evaluation

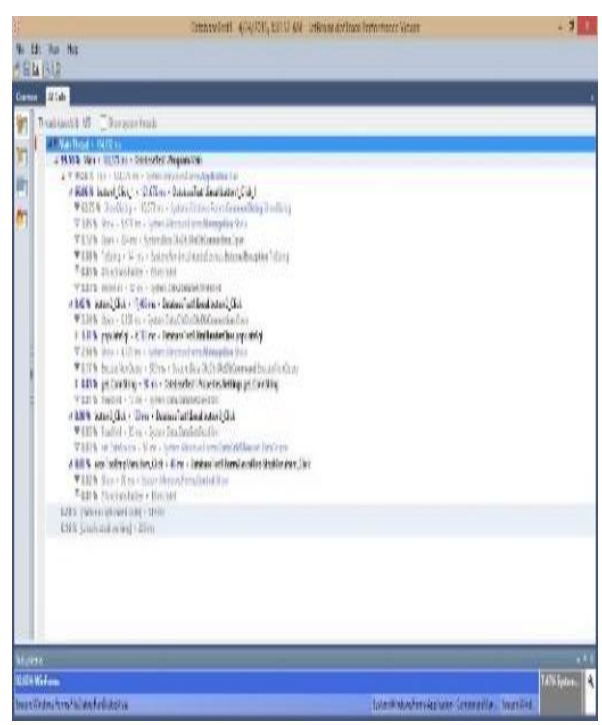

Figure- 4 System thread calls 


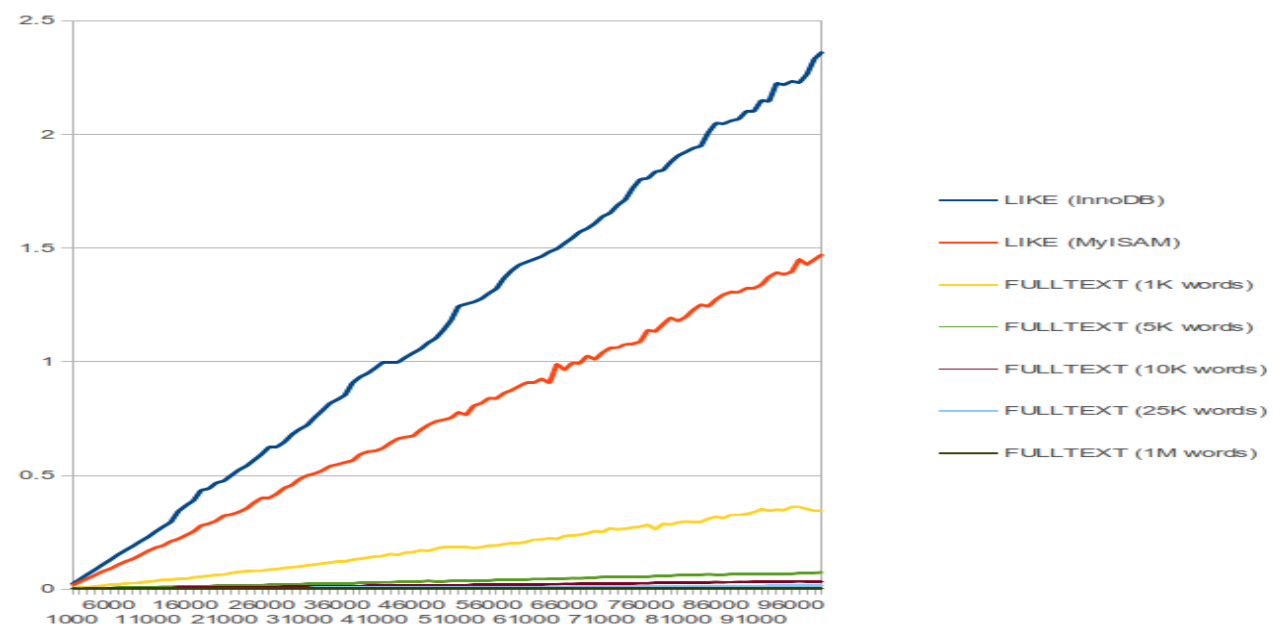

Figure-5 Comparison between full text search and like [14]

Full text search query performs better when the text data of column is low redundancy. Full text search query performance is better when a vocabulary of 1000 words to 10000 words. The educated person has a passive vocabulary is 15000 to 20000 words [14].Therefore full text search query works very good for natural language. The full text search query performance is better as compare of like when the millions of records are there.

\section{CONCLUSION AND FUTURE SCOPE}

In this research paper full text indexing will solve the problem, the searching of text data of the columns for specific words in sql databases. It can search the text data using FREETEXT and CONTAINS with boolean operators. Full text search query is better than like transact sql query. Our proposed framework work on free text search query which efficiently eliminates the limitations of the existing techniques like transact sql query. In future we are using limit search results with rank in-built function in full text search [15]. For example google search engine searches the result on the basis of ranking algorithm.

\section{REFERENCES}

[1] P.Sunanda and A.Vineela International Journal of Electronics Communication and Computer Technology Volume 4 Issue 5 (September 2014)

[2] Yi Chen, Wei Wang, Ziyang Liu and Xuemin LinKeyword Search on Structured and SemiStructuredDatahttp://www.cse.unsw.edu.au/ weiw/fil es/SIGMOD09-KeywordSearch-Final.pdf

[3] Pankaj Arora, Rubal Chaudhry Wadhawan, Er. Satinder Pal AhujaInternational Journal of Advanced Research in Computer Science and Software EngineeringVolume 2, Issue 1, January 2012 ISSN: $2277128 \mathrm{X}$

[4] Rajesh Piplode, Umesh Kumar Singh International Journal of Advanced Research in Computer Science and Software Engineering Volume 2, Issue 9, September 2012 ISSN: 2277 128X
[5] Kevin hamlen, Murat kantarcioglu, latifur khan and BhavanithuraisinghamSecurity issues for cloud computing

[6] Joseph Khoury How is it made? Google Search Engine.

[7] SoumyaSen, Ranak Ghosh, Debanjali Paul, NabenduChakiInternational Journal of Software Engineering \& Applications (IJSEA)Vol.3, No.1, January 2012

[8] Vandana BirleInternational Journal of Advanced Research in Computer Science and Software Engineering

[9] Volume 2, Issue 11, November 2012 ISSN: 2277 128XMajid Khan and M. N. A. Khan, SZABISTExploring Query Optimization Techniques in Relational Databases. International Journal of Database Theory and Application

[10] Manish Sharma, Atul Chaudhary and Santosh Kumar Query Processing Performance and Searching over Encrypted Data by using an Efficient AlgorithmInternational Journal of Computer Applications

[11] Imran SarwarBajwa, RiazUl Amin, M. Asif Naeem, M. Abbas Choudhary [2006]"Speech Language Processing Interface for Object-Oriented Application Design using a Rule-based Framework", In International Conference on Computer Applications, (ICCA 2006) Yangon, Myanmar, pp. 323-329

[12] Microsoftfulltextsearchhttps://msdn.microsoft.com/en IN/library/ms142571.aspx

[13] Boolean operator in full text search queryhttps://msdn.microsoft.com/enus/library/ms1425 83.aspx\#Using_Boolean_Operators

[14] http://makandracards.com/makandra/12813performance-analysis-of-mysql-s-fulltext-indexesandlike-queries-for-full-text-search [15]Limit search result withrankhttps://msdn.microsoft.com/enIN/library/cc8 79245.aspx 